\title{
Radiation dose audit at Hampshire Hospitals NHS Foundation Trust's computed tomography coronary angiography clinical service
}

\section{(D) Vilim Kalamar*, (DPeter Chapman, (D) Julian Elford, (D) Jade Fleet, (i)Alison Wright}

Hampshire Hospitals NHS Foundation Trust, Winchester, United Kingdom
RECEIVED:

October 21, 2018

ACCEPTED:

November 5, 2018

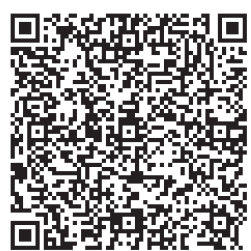

KEYWORDS: computed tomography, cardiology, radiology, dose. CITATION: Cardiol Croat. 2018;13(11-12):409-410. | https://doi.org/10.15836/ccar2018.409

*ADDRESS FOR CORRESPONDENCE: Vilim Kalamar, Hampshire Hospitals NHS Foundation Trust, Department of Radiology, S022 5DG Winchester, United Kingdom. / Phone: +447400303314 / E-mail: vilimkalamar@hotmail.com ORCID: Vilim Kalamar, https://doi.org/0000-0002-1432-9275 • Peter Chapman, https://doi.org/0000-0002-2200-1835 Julian Elford, https://doi.org/0000-0002-3750-0354 • Jade Fleet, https://doi.org/0000-0003-4315-7801 Alison Wright, https://doi.org/0000-0002-8918-4613

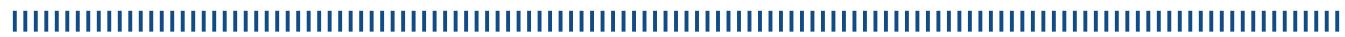

Objective: Coronary artery disease (CAD) is one of the major public health problems in the world today. The updated 2016 National Institute for Health and Care Excellence guidelines are notable for their new suggested use of computed tomography coronary angiography (CTCA) as the first-line investigation in all patients with stable chest pain. ${ }^{1,2}$ With the increasing emergence of CTCA as a routine outpatient investigation, awareness of patient exposure to ionising radiation has been identified It is the aim of this first ever CTCA radiation dose exposure audit at Hampshire Hospitals NHS Foundation Trust (HHFT) to set a benchmark for which we can compare all our future CTCAs against and to strive for increased patient safety by implementing methods to systematically reduce radiation doses without compromising diagnostic image quality.

Material and Methods: A retrospective analysis of all patients attending HHFT between 2013 to 2016 for a clinically indicated CTCA was performed. We had a total of 39 subjects. The total Dose-Length Product (DLP) and heart rate at time of image acquisition of each scan was used for analysis. Their averages were calculated by adding each of their respective totals from each individual scan and dividing them by the total number of scans performed.

Results: In the scans that we performed, the mean total DLP was 177.26mGy (Figure 1). Non-specifically, the Royal College of Radiology (RCR) states the following: the radiation dose should be as low as possible, commensurate with diagnostic image quality. ${ }^{3}$ The mean heart rate of our subjects at time of image acquisition was $59 \mathrm{bpm}$ (beats per minute) (Figure 2). Specifcally, the RCR's guidelines suggest that the patient's heart rate should be <65bpm during the scan. ${ }^{3}$ We successfully achieved this.

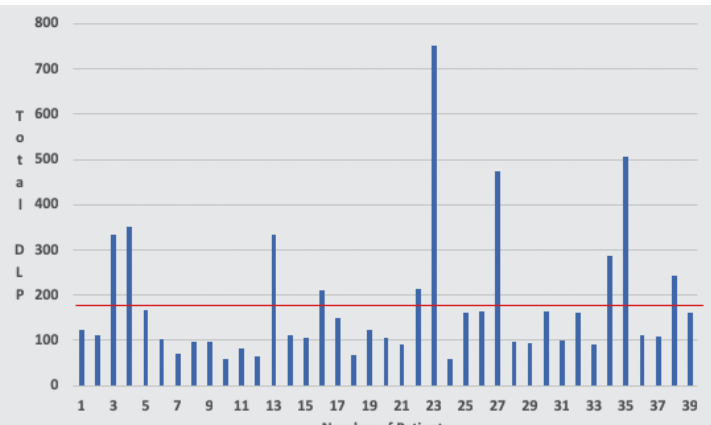

FIGURE 1. We plotted each of our 39 subject's radiation dose exposures on this graph. Each separate subject is placed along the $x$-axis. The level of radiation dose exposure expressed as milligray ( $m G y$ ) is depicted on the $Y$-axis. The red line represents the mean total dose length product (DLP) exposure (177.26mGy).

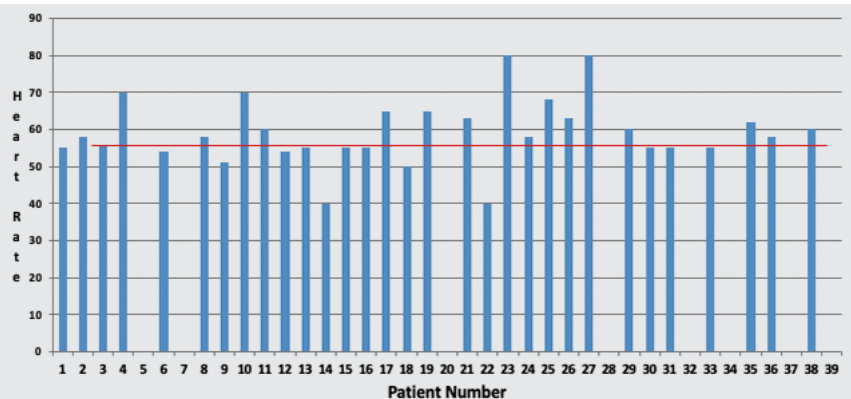

FIGURE 2. We plotted each of our 39 subject's heart rates during their scan. Each separate subject is placed along the $\mathrm{x}$-axis. The heart rate expressed in beats per minute (bpm) is placed along the $Y$-axis. The red line represents the mean heart rate of all subjects at time of image acquisition $(59 \mathrm{bpm})$. 


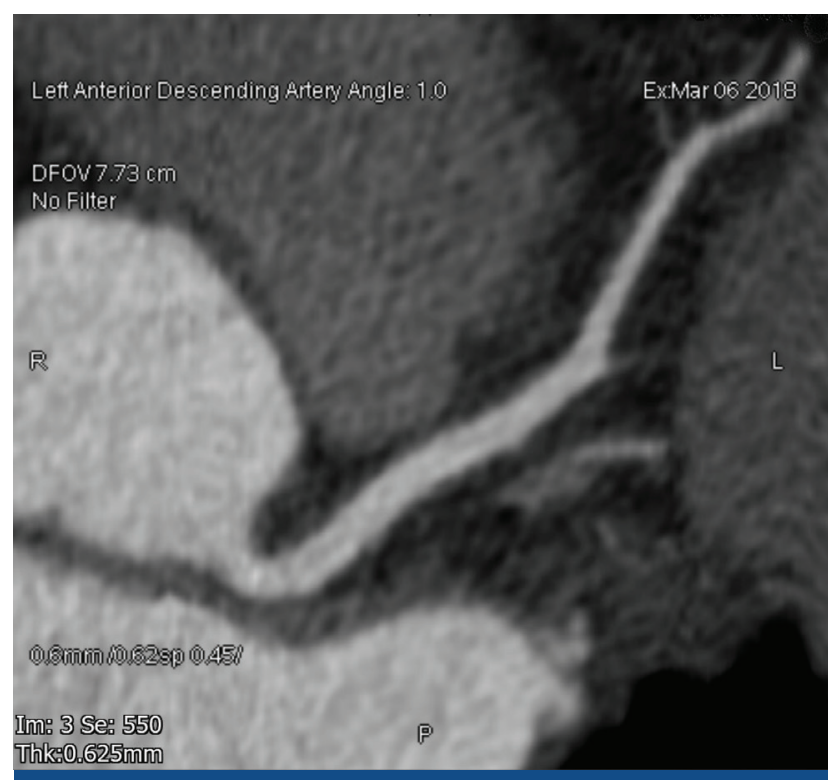

FIGURE 3. This represents a multi-planar reconstructed image depicting a patent, non-calcified left anterior descending artery in one of our subjects.

Conclusion: HHFT have just begun a regular and consistent CTCA service. It is imperative to undertake calculated measures to methodically reduce patient exposure to ionising radiation, thus concomitantly prioritising patient safety, without compromising diagnostic image quality. The CTCA has traditionally been labelled as a high radiation dose investigation which has led to concerns and potential reluctance for clinicians to incorporate it in their clinical service. However, achieving optimal image quality (Figure 3) at the lowest doses can be challenging. ${ }^{4} \mathrm{CTCA}$ staff at HHFT have collaborated and discussed internally to identify some strategies and steps towards dose reduction. This first audit loop will serve as our Trust's benchmark-a specific dose value and heart rate at time of image acquisition to work towards reducing.

1. Moss AJ, Williams MC, Newby DE, Nicol ED. The Updated NICE Guidelines: Cardiac CT as the First-Line Test for Coronary Artery Disease. Curr Cardiovasc Imaging Rep. 2017;10(5):15. https://doi.org/10.1007/s12410-017-9412-6

2. National Institute for Health and Clinical Excellence. Chest pain of recent onset: assessment and diagnosis. Available from: https://pathways.nice.org.uk/pathways/chestpain\#path=view\%3A/pathways/chest-pain/assessing-and-diagnosing-suspected-stable-angina.xml\&content=view-node\%3Anodes-diagnostic-investigations (0ct 21, 2018).

3. The Royal College of Physicians, the British Society of Cardiovascular Imaging and The Royal College of Radiologists. Standards of practice of computed tomography coronary angiography (CTCA) in adult patients. Available from: https://www.rcr.ac.uk/system/files/publication/field_publication_files/BFCR14\%2816\%29_CTCA.pdf (0ct 21, 2018).

4. White SK, Castellano E, Gartland N, Patel T, Padley SP, Rubens MB, et al. Quality assurance in cardiovascular CT: a practical guide. Clin Radiol. 2016 Aug;71(8):729-38. https://doi.org/10.1016/j.crad.2016.01.024 